\title{
Über Gebilde, die aus Tetraedern und Flächen zweiter Klasse zusammengesetzt sind.
}

Von W. Franz Meyer in Königsberg i. P.

In einer voraufgehenden Note hat Herr V. Eberhard einige bemerkenswerte Sätze über zwei Tetraeder aufgestellt und zum Teil bewiesen.

Im folgenden sollen diese Sätze und verwandte mit algebraischen Hilfsmitteln behandelt werden, wodurch, wie ich meine, nicht nur der gegenseitige Zusammenhang der Sätze schärfer hervortritt, sondern auch eine große Reihe von Verallgemeinerungen sich fast von selbst aufdrängt.

Als Kern des Ganzen erscheint eine Identität, die die Geraden (Kanten) zweier beliebiger Tetraeder miteinander verknüpft, d. h., es gibt eine Simultaninvariante beider Tetraeder, deren identisches Verschwinden aussagt, daß, wenn sich fünf Paare entsprechender Kanten beider Tetraeder treffen, dies auch für das sechste Paar stattfindet. Diese Simultaninvariante nimmt die einfachste Gestalt an, wenn die beiden Tetraeder als dualistisch einander gegenüberstehend aufgefaßt werden, und ist auch einer metrischen Deutung fähig.

Die gemeinte Identität bleibt gültig, wenn eines der Tetraeder oder auch beide in ebene Vierecke ausarten, und es wird erst dann notwendig, sie durch eine wesentlich andere Identität zu ersetzen, wenn auch die Ebenen beider ebener Vierecke so zusammenfallen, daß ihre Schnittachse unbestimmt wird.

\section{\$ 1. Über das aus einer Fläche zweiter Klasse, einem Tetraeder und einem Punkte zusammengesetzte Gebilde.}

Das Tetraeder $\mathrm{T}$ mit den Ecken $A_{i}$ und den Ebenen $\boldsymbol{\alpha}_{i}$ (wo der Index $i$ die Werte $1,2,3,4$ oder auch $i, k, l, m$ durchläuft) werde als Koordinatentetraeder gewählt. Ferner sei gegeben eine vorderhand ganz beliebige Fläche zweiter Klasse (oder ein EbenenPolarsystem) $\Gamma$ :

$$
\Gamma=\Sigma \Sigma c_{i k} u_{i} u_{k}=0,\left(c_{i k}=c_{k i}\right)
$$

sowie ein beliebiger Punkt $P(y)$ mit den Koordinaten $y_{i}$. 
Durch den Punkt $P$ lege man jeweils die Gerade $p_{i}$, die zur Ebene $\alpha_{i}$ bezüglich $\Gamma^{1}$ ) konjugiert ist: die Spur dieser Geraden $p_{i}$ in der Ebene $\alpha_{i}$ sei der Punkt $Y_{i}$ mit den Koordinaten $Y_{i r}(r=i, k, l, m)$.

Der Pol der Ebene $\alpha_{i}$, der die Koordinaten $c_{i r}(i, k, l, m)$ besitzt, werde $C_{i}$ genannt, dann ist die Gerade $p_{i}$ eben die, welche $P$ mit $C_{i}$ verbindet.

Die Koordinaten der Pankte $Y_{i}$, deren Tetraeder mit $H$ bezeichnet sei, sind dargestellt durch das folgende Schema ${ }^{2}$ ), wobei der in den Hauptdiagonalgliedern auftretende Wert Null zweckmäßiger durch $c_{i i} y_{i}-c_{i i} y_{i}(i=i, k, l, m)$ ersetzt werde:

$(2)\left\{\begin{array}{cccc}Y_{i} & Y_{k} & Y_{l} & Y_{m} \\ c_{i i} y_{i}-c_{i i} y_{i}, & c_{k k} y_{i}-c_{k i} y_{k}, & c_{l l} y_{i}-c_{l i} y_{l}, & c_{m m} y_{i}-c_{m i} y_{m} \\ c_{i i} y_{k}-c_{i k} y_{i}, & c_{k l} y_{k}-c_{k l} y_{k}, & c_{l l} y_{l}-c_{l k} y_{l}, & c_{m m} y_{k}-c_{m k} y_{m} \\ c_{i i} y_{l}-c_{i 2} y_{i} & c_{k k} y_{l}-c_{l l t} y_{k}, & c_{l l} y_{l}-c_{l i} y_{l}, & c_{m m} y_{l}-c_{m b} y_{m} \\ c_{i i} y_{m}-c_{i m} y_{i}, & c_{k l} y_{m}-c_{k m} y_{k}, & c_{l l} y_{m}-c_{l m} y_{l}, & c_{m m} y_{m}-c_{m m} y_{m}\end{array}\right.$

oder auch, in eine einzige Formel zusammengezogen:

$$
Y_{r s}=c_{r r} y_{s}-c_{r s} y_{r}(r, s=i, l, l, m) \text {. }
$$

Man lege jetzt etwa durch die Ecke $A_{m}$ ron T die zur Ecke $\left(Y_{i}, Y_{k}, Y_{l}\right)$ konjugierte Gerade $g_{m}$. Die Koordinaten der Ebene $\left(Y_{i}^{\prime}, Y_{k}, Y_{l}\right)$ oder kürzer $v_{m}$ seien mit $v_{m r}(r=i, k, l, m)$, der Pol dieser Ebene mit $V_{m}$ bezeichnet. Dann besitzen die Koordinaten $V_{m r}(r=i, k, l, m)$ von $V_{m}$ die Werte:

$$
V_{m r}=c_{r i} v_{m i}+c_{r i} v_{m n}+c_{r l} v_{m l}+c_{r m} v_{m m}=\sum_{s=i}^{m} c_{r s} v_{m s} .
$$

Die Gerade $g_{m}$ verbindet $A_{m}$ mit $V_{m}$ und trifft die Ebene $\alpha_{m}$ in einem Punkte $G_{m_{6}}$ mit den Koordinaten:

$$
V_{m i}, V_{m l i}, V_{m l}, 0 \text {. }
$$

Gemäß (2) sind dann die drei ersten Werte (4) dargestellt durch die Determinanten $(s=i, k, l)$ :

(5) $V_{m s}=\left|\begin{array}{llll}c_{i i} y_{i}-c_{i i} y_{i}, & c_{k k} y_{i}-c_{k i} y_{k}, & c_{l l} y_{i}-c_{l i} y_{l}, & c_{s i} \\ c_{i i} y_{k}-c_{i k} y_{i}, & c_{k k} y_{k}-c_{k k} y_{k}, & c_{l l} y_{l}-c_{l k} y_{l}, & c_{s k} \\ c_{i i} y_{l}-c_{i l} y_{i,} & c_{k i} y_{l}-c_{k l} y_{k}, & c_{l l} y_{l}-c_{l l} y_{l}, & c_{s l} \\ c_{i i} y_{m}-c_{i m} y_{i}, & c_{k k} y_{m}-c_{k m} y_{k}, & c_{l l} y_{m}-c_{l m} y_{l}, & c_{s m}\end{array}\right|$.

1) Der Zusatz „bezüglich $\Gamma^{\prime \prime}$ werde im folgenden als selbstverständlich zumeist weggelassen.

2) S. auch meine demnächst erscheinende Abhandlung im Archiv für Math. und Phys. (3), Bd. XI. Die den homogenen Koordinaten (2) der Punkte $Y_{i}$ zugehörigen Proportionalitätsfaktoren werden, da sie nicht weiter in Betracht kommen, unterdriickt. 
Setzt man zur Abkürzung:

$$
\gamma_{m}=\left|y C_{i} C_{k} C_{l}\right| \text {, }
$$

d. h. gleich der aus den Koordinaten der Punkte $(y), C_{i}, C_{k}, C r$ gebildeten Determinante, so reduzieren sich die drei Werte (5) nach einfachen Determinantensätzen auf die folgenden:

$$
V_{m i}=c_{i i} y_{k} y_{l} \gamma_{m}, V_{m i}=c_{k k} y_{i} y_{l} \gamma_{m}, V_{m l}=c_{l l} y_{i} y_{k} \gamma_{m} .
$$

Demnach lassen sich die Koordinaten (4) des Punktes $G_{m}$, der Geraden $g_{m}$ in der Ebene $\alpha_{m}$, auch in der Gestalt schreiben :

$$
c_{i i} y_{k} y_{l} y_{m}, c_{k k} y_{i} y_{l} y_{m}, c_{l l} y_{i} y_{k} y_{m}, 0 \text {. }
$$

Wiederholt man nunmehr den für die Ecke $A_{m}$ ausgeführten Prozeß für die drei anderen Ecken $A_{i}, A_{k}, A_{l}$ von T, so geht aus (8) hervor, daß sich die vier Geraden $g_{i}$ in einem Punkte treffen, nämlich in einem Punkte $Q(z)$ mit den Koordinaten:

$$
\begin{aligned}
z_{i}: z_{k}: z_{l}: z_{m} & =c_{i i} y_{k} y_{l} y_{m}: c_{k k} y_{i} y_{l} y_{m}: c_{l l} y_{i} y_{k} y_{m}: c_{m m} y_{i} y_{k} y_{l} \\
& =\frac{c_{i i}}{y_{i}}: \frac{c_{k k}}{y_{k}}: \frac{c_{l l}}{y_{l}}: \frac{c_{m m}}{y_{m k}} .
\end{aligned}
$$

Dieses Erg'ebnis umfaßt zwei Sätze, von denen der eine nur die Tatsache im Auge hat, daß sich die vier Geraden $g_{i}$ überhaupt in einem Punkte treffen, während der andere die genauere Abhängigkeit dieses Treffpunktes $Q(z)$ von dem Ausgangspunkte $P(y)$ verfolgt.

Der erste Satz lautet:

I. "Legt man durch einen Punkt $P$ die vier Geraden $p_{i}(i=i, k, l, m)$, die resp. zu den Ebenen $\alpha_{i}$ eines Tetraeders $\mathrm{T}$ mit den Ecken $A_{i}$ konjugiert sind in be$z u g$ anf eine beliebige Fläche zweiter Klasse $\Gamma$, bezeichnet die Spuren der Geraden $p_{i}$ in den Ebenen $\alpha_{i}$ mit $Y_{i}$, die vier Ebenen $\left(Y_{k} Y_{l} Y_{m}\right)$ mit $v_{i}$ und legt wiederum dureh die Ecken $A_{i}$ von $T$ die vier Geraden $g_{i}$, die zu den Ebenen $v_{i}$ konjugiert sind in bezug auf $\Gamma$, so schneiden sich die vier Geraden $g_{i}$ in einem und demselben Punkte $Q$.

Die Beziehung der beiden Punkte $P, Q$ ist eine gegenseitige, so dabmanvermöge der nämlichen Konstruktion von $Q$ wieder zu $P$ gelangt."

Hieraus geht der erste Satz des Herrn Eberhard hervor, wenn $\Gamma=0(1)$ im besonderen die Gleichung des $\mathrm{Kugelkre}$ ises ist; die Geraden $p_{i}$ stehen dann senkrecht auf den Ebenen $a_{i}$ und die Geraden $g_{c}$ senkrecht auf den Ebenen $v_{i}$.

Variiert man nunmehr den Punkt $P(y)$ im Raume, so entspricht ihm jeweils ein Punkt $Q(z)$ vermöge der Transformation (I). 
Von dieser eineindeutigen, involutorischen, kubischen Transformation - die kurz mit $T$ bezeichnet sei - mögen zunächst die wesentlichsten bekannten Eigenschaften ${ }^{1}$ ) zusammengestellt werden.

Durch $T$ werden je zwei Punkte $P, Q$ einander zugeordnet, die konjugiert sind in bezug auf alle Flächen zweiter Ordnung desjenigen Netzes $N$, dessen acht Grundpunkte die Koordinaten $\pm \sqrt{c_{i i}}, \pm \sqrt{c_{k k}}, \pm \sqrt{c_{l l}}, \pm \sqrt{c_{m m}}$ (bei allen Kombinationen der Wurzelvorzeichen) besitzen ; diese Punk te sind zugleich die "Einheitspunkte", das sind die sich selbst entsprechenden Punkte der Transformation $T$.

Das Bild einer Ebene ist eine Fläche dritter Ordnung mit vier Knotenpunkten in den Ecken $A_{i}$ von $\mathrm{T}$ und umgekehrt; das Bild einer. Geraden ist eine, durch die Ecken $A_{i}$ gehende kubische Raumkurve und umgekehrt.

Übt man auf einen Punkt $(y)$ zunächst die spezielle Kollineation $K$ aus:

$$
\text { K) } y_{i}^{\prime}=\frac{y_{i}}{c_{i i}}
$$

sodann auf den Punkt $y^{\prime}$ die spezielle Transformation $T^{\prime \prime}$ :

$$
\left.T^{\prime}\right) \quad z_{i}=\frac{1}{y_{i}^{\prime}},
$$

so entsteht die Transformation $T$; man sagt, $T$ entsteht durch $\mathrm{Zu-}$ sammensetzung ron $K$ und $T^{\prime \prime}$ :

$$
T=K T^{\prime}=T^{\prime \prime} K
$$

wobei die Reihenfolge der Zusammensetzung gleichgültig ist. $K$ ist diejenige Kollineation, die die Ebenen (und damit zugleich die Ecken) von T' fest läßt, und zugleich den Punkt $C$ mit den Koordinaten $c_{i i}, c_{k / 7}, c_{l l}, c_{m m}$, als Punkt $y$ aufgefaßt, in den ${ }_{\text {Ein- }}$ heitspunkt" $(1,1,1,1)$, als Punkt $y^{\prime}$ aufgefaßt, überführt.

$T^{\prime}$ ist diejenige Transformation (I), die entsteht, wenn die vier Koeffizienten $c_{i i}$ in der Gleichung (1) $\Gamma=0$ der Fläche zweiter Klasse $\Gamma$ der Einheit proportional werden; dies tritt $u$. a. ein, wenn $\Gamma=0$ die Gleichung des Kugelkreises ist.

Die Einheitspunkte von $T^{\prime}$ sind dann die acht "Einheitspunkte" des Koordinatentetraeders $\mathrm{T}$ (deren Koordinaten +1 oder - 1 sind), oder geometrisch, die Mittelpunkte der acht dem Tetraeder ' $T$ einbeschriebenen Kugeln. Vermöge $T^{\prime}$ entspricht der unendlich fernen Ebene diejenige Fläche dritter Ordnung, die zugleich als Ort eines Punktes $P$ entsteht, für den die Fußpunkte der von ihm auf die Ebenen $\alpha_{i}$ von $\mathrm{T}$ gefällten Lote in einer Ebene liegen.

1) Siehe meine Arbeiten im Archiv für Math. u. Phys. (3), Bd. V, p. 168, Bd. VII, p. 135, Bd. XI. 
Wir fassen alles dies kurz zusammen in dem Satze:

II. "Die Punkte $P(y), Q(z)$ des Satzes I entsprechen einander vermöge der eineindeutigen, involutorischen, kubischen Transformation $T(I)$ mit den soeben aufgezählten Eigenschaften."

Hieraus geht der dritte Satz des Herrn Eberhard hervor, wenn man von vornherein $\Gamma=0$ (1) als Gleichung des Kugelkreises zu Grunde legt; $T$ reduziert sich dann auf $T^{\prime \prime}$ and $K$ auf die identische Kollineation.

Als die einfachste geometrische Charakterisierung der Transformation $T$ (I) dürfen wir gerade die durch den Satz $\mathbf{I}$ angegebene Eigenschaft von $T$ ansprechen. Von dieser aus gelangt man aber sofort zu weiteren Eigenschaften, die, mit anderen, früher ${ }^{1}$ ), von mir aufgestellten, kombiniert, ein einigermaßen vollständiges Bild von dieser wichtigen Transformation zu entwerfen gestatten.

Die Vergleichung von (I) mit (1) lehrt sofort, daß bei festgehaltenem Punkte $P(y)$, der Punkt $Q(z)$ (I) unverändert bleibt, wenn auch in (1) die Koeffizienten $c_{i k}$ mit zwei ungleichen Indices willkürlich gelassen werden. An die Stelle der ursprünglichen Fläche zweiter Klasse $\Gamma$ tritt also eine ganze $\infty^{6}$ lineare Schar $\Sigma$ solcher, die sich dadurch charakterisieren läßt, daf ihr einmal die spezielle Fläche

$$
\Gamma^{\prime} \equiv \Sigma c_{i i} w_{i}^{2}=0
$$

die $\mathrm{T}$ als Poltetraeder besitzt, angehört, anderseits die Gesamtheit der $\mathrm{T}$ einbeschriebenen Flächen zweiter Klasse.

Indessen läßt sich diese Schar $\Sigma$ von Flächen zweiter Klasse noch einfacher festlegen. Das Netz $N$ von Flächen zweiter Ordnung, durch das oben die Transformation $T$ charakterisiert wurde, ist, wenn $\lambda_{k}, \lambda_{l}, \lambda_{n}$ drei homogene Parameter bedeuten, dargestellt durch:

$$
N \equiv \lambda_{k i}\left(x_{i}^{2} c_{k l}-x_{k}^{2} c_{i i}\right)+\lambda_{l}\left(x_{i}^{2} c_{l l}-x_{k}^{2} c_{i i}\right)+\lambda_{m}\left(x_{i}^{2} c_{m m}-x_{m}^{2} c_{i i}\right)=0 .
$$

Demnach ist die $\infty^{6}$ lineare Sebar $\Sigma$ von Flächen zweiter Klasse $\Gamma$ (1) bei willkürlichen $c_{i k}(i=\mid=k)$, vermöge deren einem Punkte $P(y)$ stets ein und derselbe Punkt $Q(z)$ zugeordnet wird, gerade die zum Netze $N(13)$ apolare ${ }^{2}$ ) Schar."

Insbesondere gehören also der Schar $\Sigma$ die acht doppelt zo zählenden Einheitspunkte der Transformation $T$ an, und umgekehrt

1) S. die Fußnote auf p. 4.

2) Eine Fläche zweiter Ordnung and eine Fläche zweiter Klasse heißen apolar, wenn ihre bilineare Invariante verschwindet. 
ist durch jene acht Einheitspunkte die Schar $\Sigma$ bestimmt, ${ }^{1}$ ) wie auch analytisch leicht $\mathrm{zu}$ bestätigen ist.

Der $\infty^{6}$ Schar $\Sigma$ gehört eine $\infty^{5}$ - nicht lineare - Schar von in einen Klassenkegelschnitt $K_{9}$ ausgearteten Flächen an. In jeder Ebene $E$ liegt noch eine $\infty^{2}$ - wieder lineare - Schar solcher $K_{2}$; zu dieser ist in $E$ eine $\infty^{2}$ lineare Schar von Ordnungskegelschnitten $C_{2}$ apolar, und diese Schar von $C_{2}$ wird aus $E$ durch das Flächennetz $N(13)$ ausgeschnitten.

Die in bezug auf eine solche $\infty^{2}$ Schar von $C_{\mathrm{g}}$ (in der Ebene $E$ gelegenen) konjugierten Panktepaare $(P, Q)$ erfüllen eine Kurve dritter Ordnung $C_{3}$, und diese wird aus $E$ gerade durch diejenige Fläche dritter Ordnung ausgeschnitten, die der Ebene $E$ vermöge der Transformation $T$ entspricht. ${ }^{2}$ )

Es gibt im ganzen acht Flächen zweiter Ordnung, die einen solchen Kegelschnitt $C_{2}$ enthalten und zugleich dem Tetraeder T einbeschrieben sind; die acht Pole dieser Flächen bezüglich der Ebene $E$ sind stets die acht Einheitspunkte der Transformation $T$.

Die bisher betrachtete Figur des Tetraeders T, der Fläche zweiter Klasse $\Gamma$, und des - variierend gedachten - Punktes $P(y)$, sowie des jeweils dadurch bestimmten, dem Tetraeder T einbeschriebenen Tetraeders $H$, der Punkte $\vec{Y}_{i}$ und des Punktes $Q(z)$, läft sich indessen noch auf andere Weise variieren.

Es werde jetzt außer T noch der Punkt $P(y)$ und das Tetraeder $H$ der Punkte $Y_{i}$ festgehalten, so zwar, daß die vier Punkte $Y_{i}$ willkürlich auf den Ebenen $\alpha_{i}$ von $\mathrm{T}$ gewählt seien; hingegen werde eine zugehörige Fläche zweiter Klasse $\Gamma$ (1) gesucht.

Aus dem Schema (2) geht hervor, dab dann den neun Koeffizientenverhältnissen der $c$ in (1) acht lineare - ersichtlich linear nabhängige - Bedingungsgleichungen auferlegt werden; geometrisch sagen dieselben aus, daf die vier Pole $C_{i}$ der Ebenen $\alpha_{i}$ von T bezüglich $\Gamma$ stets auf vier gegebenen Geraden liegen müssen.

Es gibt also noch eine $\infty^{1}$ lineare Schar von Flächen zweiter Klasse $\Gamma$, die der Aufgabe genügen. Die Formeln (I) zeigen alsdann, dal der einem beliebig gegebenen Punkte $P(y)$ zugeordnete Punkt $Q(z)$ eine Gerade $g$ beschreibt. $\left.{ }^{3}\right)$ Seien $c_{i k}, c_{i k}^{\prime}$ die Koef-

1) Zwischen den acht Quadraten der Linearformen (in den $u_{i}$ ), deren Verschwinden die acht Yinheitspunkte darstellt, findet danach eine lineare Identität statt; das sagt aus, daf durch sieben derselben der achte eindeutig bestimmt ist. Dies ist ersichtlich nur ein besonderer Fall eines bekannten Satzes über die acht Grundpunkte eines beliebigen Flächennetzes zweiter Ordnung.

2) S. meine Abhandlung, Archir der Math. und Phys. (3), Bd. XI.

${ }^{3}$ ) In der $\infty^{1}$ Schar von Flächen $\Gamma$ gibt es vier, von denen je eine Ebene $\alpha_{i}$ von $T$ berïhrt. Diese vier Berührungspunkte sind gerade die vier Schnittpunkte von $g$ mit den Ebenen $\alpha_{i}$. Da es in einer linearen Schar von Flüchen zweiter Klasse vier in Klassenkegelschnitte $K_{2}$ ausgeartete Flächen gibt, so existieren, wenn einer dieser $K_{2}$ im besonderen der Kugelkreis ist, wenn also die $Y_{i}$ die Fußpunkte der von $P$ auf die ai gefällten Lote $p_{i}$ sind, noch drei $K_{2}$, in bezug auf welche die $p_{i}$ ebenfalls $z u$ den $\alpha_{i}$ konjugiert sind. 
fizienten für irgend zwei Flächen der Schar, $\lambda, \lambda^{\prime}$ zwei homogene Parameter, so ist die Gerade $g$ repräsentiert durch die Gleichungen

$$
z_{i}=\frac{\lambda c_{i i}+\lambda^{\prime} c^{\prime}{ }_{i i}}{y_{i}}
$$

Bezeichnet man mit $p_{i k}$ die Strahlenkoordinaten von $g$, und setzt zur Abkürzung:

$$
q_{i k}=\left|\begin{array}{cc}
c_{i i} & c_{i i}^{\prime} \\
c_{k k} & c_{k k}^{\prime}
\end{array}\right|
$$

so sind die $p_{i k}$ bestimmt durch:

$$
p_{i k} y_{i} y_{k}=q_{i k} \text {. }
$$

Demnach erfüllen die sämtlichen Punkte $P(y)$ des Raumes einen quadratischen Komplex, dessen Gleichung lautet:

$$
\left|\begin{array}{llll}
p_{12} & p_{34}, & q_{12} & q_{34} \\
p_{13} & p_{42}, & q_{13} & q_{42}
\end{array}\right|=0
$$

Dieser Komplex enthält alle Geraden der vier Ebenen $a_{i}$ von T; er ist eindeutig bestimmt, wenn man eine einzige Gerade $g$ kennt, die ihm angehört.

Die obigen Entwicklungen - mit Ausnahme derer über die $\infty^{1}$ lineare Schar von Flächen zweiter Klasse $\Gamma^{1}$ - lassen sich auf den linearen Raum $R_{n}$ von $n$ Dimensionen übertragen; die Fundamentalformel (I) lautet dann:

$$
z_{i}=\frac{c_{i i}}{y_{i}}(i=1,2 \ldots n+1) .
$$

Endlich bleibt die Ableitung, die zu der Formel (I) führte, unberührt, auch wenn das durch die Fläche $\Gamma$ (1) bestimmte EbenenPolarsystem $\Sigma \Sigma u_{i} v_{k} c_{i k}=0\left(c_{i k}=c_{k i}\right)$ ersetzt wird durch die umfassendere Ebenen-Korrelation $\left(c_{i k} \gtreqless c_{k i}\right)$, wo die 15 Verhältnisse der $c_{i k}$ beliebig gewählte Größen sein können. Und entsprechend wieder im $R_{n}$.

Die zu den bisher ausgesprochenen Sätzen dualistischen verstehen sich von selbst.

Die Übertragungen der bisherigen Sätze auf die nichteuklidische Geometrie eines $R_{3}$ resp. $R_{n}$ liegen auf der Hand, sobald man $\Gamma$ (1) als absolute Fläche des betreffenden Raumes auffaßt. Die Geraden $p_{i}, g_{i}$ werden dann die nichteuklidischen Senkrechten zu den Ebenen $\alpha_{i}$ resp. $v_{i}$. Endlich erkennt man auch die Erweiterung auf eine "Übernichteuklidische Geometrie",

1) In der Tat erkennt man schon im Falle des $R_{4}$, daß bei beliebig gegebenen $Y_{i}(i=1,2,3,4,5)$ den 15 bomogenen $c_{i k}$ der entsprechenden Gleichung $\Gamma$ (1) 15 lineare Bedingungen aufzuerlegen wären, daß also eine zugehörige $\Gamma$ im allgemeinen nicht mehr existiert. 
wenn man die absolute Fläche $\Gamma$ (1) ersetzt durch eine Ebenenkorrelation $\left(c_{i k} \gtreqless c_{k i}\right)$, also auch den absoluten Kegelschnitt in irgend einer Ebene $E$, durch eine gewisse Geradenkorrelation.

Es scheint nicht viele Sätze der gewöhnlichen Geometrie zu greben, die einer derartigen Verallgemeinerung fähig sind.

\section{\$ 2. Über das aus einer Fläche zweiter Klasse und aus zwei Tetraedern bestehende Gebilde.}

Die beiden in $\S 1$ betrachteten Tetraeder $\mathrm{T}\left(A_{i}, a_{i}\right)$ and $H$ $\left(Y_{i}, v_{i}\right)$ stehen in der Beziehung, daß stets die Kante $\left(\alpha_{i}, \alpha_{k}\right)$ von $\mathrm{T}$ konjugiert ist [bezüglich der Fläche zweiter Klasse $\Gamma$ (1)] zur Kante $\left(Y_{i}, Y_{k}\right)$ von $H$.

Denn da $C_{i}, C_{k}$ die Pole von $\alpha_{i}, \alpha_{k}$ sind, so ist $\left(C_{i}, C_{k}\right)$ die reziproke Polare der Kante $\left(\alpha_{i}, a_{k}\right)$.

Nun ist die Gerade $p_{i}$ konjugiert zur Ebene $\alpha_{i}, d$. h. jede Ebene durch $p_{i}$ geht durch den Pol $C_{i}$ von $\alpha_{i}$, und desgleichen geht jede Ebene durch $p_{k}$ dnrch den Pol $C_{k}$ von $\alpha_{k}$. Mithin liegt die Gerade $\left(C_{i} C_{k}\right)$ in der durch $P$ gehenden Ebene $\left(p_{i}, p_{k}\right)$, die anderseits die Gerade $\left(Y_{i}, Y_{k}\right)$ enthält. Somit trifft die Gerade $\left(Y_{i}, Y_{k}\right)$ die Gerade $\left(C_{i}, C_{k}\right)$, die reziproke Polare von $\left(\alpha_{i}, \boldsymbol{\alpha}_{k}\right), \mathbf{d} . \mathbf{h}$. die Geraden $\left(\alpha_{i}, \alpha_{k}\right)$ und $\left(Y_{i}, Y_{k}\right)$ sind konjugiert.

Allgemeiner mögen jetzt zwei Tetraeder - oder besser gesagt, zwei Vierecke im Raume - T $\left(A_{i}\right)$ und $\Delta\left(D_{i}\right)$ in der Beziehung zu einander stehen, daß stets die Kante $\left(A_{i}, A_{k}\right)$ des ersten zu der „entsprechenden" Kante $\left(D_{l}, D_{m}\right)$ des zweiten konjugiert ist in bezug auf irgend eine vorgelegte Fläche zweiter Klase $\Gamma$ (1).

Dabei werde auch die Möglichkeit ${ }^{1}$ ) zugelassen, daß das eine der beiden Vierecke, etwa $\Delta$, ein ebenes ist. Es soll gezeigt werden, in Verallgemeinerung des $z$ weiten Satzes von Herrn E berhardt, der für $\Gamma$ als Kugelkreis resultiert, dab dann auch die vier Geraden $g_{i}$, von denen je eine durch eine Ecke $A_{i}$ von $\mathrm{T}$ geht und zu der $n$ entsprechenden" Ebene $\delta_{i}=\left(D_{k}, D_{i}, D_{m}\right)$ von $\Delta$ konjugiert ist, sich in einem Punkte $Q$ treffen. Ist das der Fall, so treffen sich auch reziprok die vier Geraden $p_{i}$, von denen je eine durch eine Ecke $D_{i}$ von $\Delta$ geht und zu der "entsprechenden " Ebene $\alpha_{i}\left(A_{k}, A_{l}, A_{m}\right)$ von $\mathrm{T}$ konjugiert ist, in einem Punkte $P$, und das Punktepaar $P, Q$, sowie die Geraden $p_{i}, g_{i}$ bilden dann ersichtlich eine Verallgemeinerung der in $\$ 1$ untersuchten.

Der in Rede stehende Satz, nebst seiner Umkehrung, ist geometrisch einfach za beweisen.

Es sei wiederum $C_{i}\left(c_{i i}, c_{i k}, c_{i l}, c_{i m}\right)$ der Pol der Ebene $\alpha_{i}$ von $\mathrm{T}$, oder, was auf dasselbe hinauskommt, es sei das Viereck $\left(C_{i}\right.$, $\left.C_{k}, C_{l}, C_{m}\right)$ das Polviereck von T. Das Konjugiertsein der Kanten

1) Die weitere Möglichkeit, daß auch das zweite Viereck ein ebenes ist, wird weiter unten besonders behandelt. 
$\left(A_{i}, A_{k}\right)$ und $\left(D_{l}, D_{m}\right)$ ist gleichbedeutend mit dem Treffen der Kanten $\left(C_{l}, C_{m}\right)$ und $\left(D_{l}, D_{m}\right)$ und dieses wiederum gleichbedeutend dem Treffen der Transversalen $\left(C_{l}, D_{l}\right)$ und $\left(C_{m}, D_{m}\right)$, und umgekehrt.

Die besondere Lage der beiden Vierecke $\mathrm{T}$ und $\Delta$ läßt sich daher auch dahin charakterisieren, daß sich die beiden Vierecke $\Delta$ und $\left(C_{i}, C_{k}, C_{l}, C_{m}\right)$ in perspektiver Lage befinden. Denn da sich je zwei der vier Transversalen $\left(C_{r}, D_{r}\right)(r=i, k, l, m)$ treffen, ohne daß irgend drei von ihnen einer Ebene angehören können, so gehen alle vier durch einen und denselben Punkt, and umgekehrt. Die Transversale $\left(C_{r}, D_{r}\right)$ ist aber eben die Gerade, die durch die Ecke $D_{r}$ des Viereckes $\Delta$ geht und zugleich zur Ebene $\alpha_{r}$. des Viereckes $\mathrm{T}$ konjugiert ist.

Es gelten ersichtlich wiederum die nämlichen Ausdehnungen des Satzes, wie in $\$ 1$.

Der Fall des gewöhnlichen Raumes $R_{3}$ mag zunächst weiter verfolgt werden.

Man zählt unmittelbar $a b$, daß die perspektive Lage zweier Vierecke $\Delta$ und $\left(C_{i}, C_{k}, C_{l}, C_{m}\right)$ fünf Bedingungen involviert. Anderseits erfordert das Konjugiertsein je zweier Kanten $\left(A_{i}, A_{k}\right)$ und $D_{l}, D_{m}$ ) je eine Bedingung; von diesen sechs Bedingungen mub daher ein e Folge der fünf übrigen sein, oder, was dasselbe ist, jene sechs Bedingungen müssen an eine gewisse Identität geknüpft sein.

Um diese Identität aufzustellen, empfiehlt es sich, die Pole der Ebenen $\delta_{i}$ des Viereckes $\Delta$ einzuführen. Man bezeichne den Pol der Ebene $\hat{o}_{i}=\left(D_{k}, D_{l}, D_{m}\right)$ mit $E_{i}$ und seine Koordinaten mit $e_{i}, e_{i k}, e_{i l}, e_{i m}$. Dann ist wiederum das Konjugiertsein der Kanten $\left(A_{i}, A_{k}\right)$ und $\left(D_{l}, D_{m}\right)$ äquivalent mit dem Treffen der Kanten $\left(A_{i}, A_{k}\right)$ und $\left(E_{i}, E_{k}\right)$, also mit der durch $A_{i k}$ (oder $A_{k i}$ ) angegebenen Bedingung:

$$
A_{i l} \equiv A_{k i} \equiv\left|\begin{array}{l}
e_{i l}, e_{i m} \\
e_{k l}, e_{k m}
\end{array}\right| \equiv e_{i l} e_{k m}-e_{i m} e_{h l}=0 .
$$

Von der vierreihigen Determinante der $e_{i k}$ verschwinden somit, bei perspektiver Lage der Vierecke $\Delta$ und $\left(C_{i}, C_{k}, C_{l}, C_{m}\right)$, gerade diejenigen sechs zweiten Minoren, von deren Elementen keineszwei gleiche Indices trägt; zwischen diesen Minoren selbst, bei beliebig gegebenen $e_{i k}$, muf die fragliche Identität stattfinden.

Eliminiert man, unter Bevorzugung der Index $i$, einmal aus den drei Gleichungen $A_{i k}=0, A_{i l}=0, A_{i m}=0$ die Größen $e_{i k}$, $e_{i l}, e_{i m}$, so erscheint als Resultantengleichung:

$$
e_{k l} e_{l m} e_{m k}-e_{l k} e_{m l} e_{k m}=0 .
$$

Dieselbe Gleichung (16) erhält man aber auch durch Elimination der Größen $e_{k i}, e_{l i}, e_{m i}$ aus den drei übrigen Gleichungen $A_{k l}=0, A_{l m}=0, A_{m b}=0$. 
Nunmehr operiere man mit den linken Seiten $\left.{ }^{1}\right) A_{i k}, A_{i l}, A_{i m}$; $A_{k l}, A_{l m}, A_{\eta n k}$ der Gleichungen selbst, geordnet nach den $e_{i k}, e_{i l}, e_{i m}$ resp. $e_{k i}, e_{i i}, e_{m i}$ :

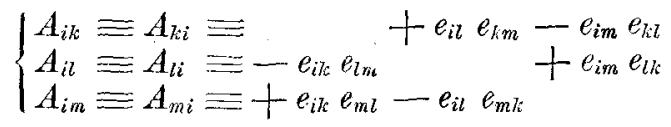

$$
\begin{aligned}
& \left\{\begin{array}{l}
A_{l m} \equiv A_{m l} \equiv+e_{k i} e_{m l}+e_{l i} e_{m b}-e_{m i} e_{l k}+e_{m i} e_{k l} \\
A_{k m} \equiv A_{m k} \equiv-e_{l i} e_{k m} \\
A_{l l} \equiv A_{k l} \equiv+e_{k i} e_{l m}-e^{2}
\end{array}\right.
\end{aligned}
$$

wo jeweils zwei Ausdrücke $A_{i k}$ und $A_{l m}$ durch Vertauschung beider Indices in jedem Faktor $e$ aus einander hervorgehen. Setzt man zur Abkürzung: ${ }^{2}$ )

$$
R \equiv e_{m l} e_{l k} e_{k m}-e_{l m} e_{m k} e_{k l},
$$

und multipliziert die drei Ausdrücke $(17 a)$ resp. mit $e_{m k} e_{l k}, e_{m k} e_{k l}$, $e_{k m} e_{l k}$, anderseits die drei Ausdrücke $(17 b)$ resp. mit $e_{k m} e_{k i}$, $e_{k m} e_{k k}, e_{m k} e_{k l}$, und addiert beidemal, so kommt

$$
\left\{\begin{array}{l}
A_{i k} e_{m k} e_{l k}+A_{i l} e_{m k} e_{k l}+A_{i m} e_{k m} e_{l i}=e_{i k} R \\
A_{l m} e_{k m} e_{k l}+A_{k m} e_{k m} e_{i k}+A_{l l} e_{m l i} e_{k l}=-e_{k i} R,
\end{array}\right.
$$

und damit, durch Elimination von $R$, die gewünschte Identität, zunächst in der Gestalt:

$$
\begin{aligned}
{[k, i] } & \equiv e_{k i}\left\{A_{i k} e_{m k} e_{l k}+A_{i l} e_{m k} e_{k l}+A_{i m} e_{k m} e_{l k}\right\} \\
& +e_{i k}\left\{A_{i m} e_{k m} e_{k l}+A_{k m} e_{k m} e_{l k}+A_{l k} e_{m k} e_{k l}\right\} \equiv 0
\end{aligned}
$$

Diese läht sich sofort, durch blobe Gliederamstellung, auch in die folgenden Gestalten bringen:

$$
\begin{aligned}
& {[k, l] \equiv e_{k l}\left\{A_{l i} e_{k i} e_{m k}+A_{l k} e_{i k} e_{m k}+A_{l m} e_{i k} e_{k m}\right\}} \\
& +e_{l k}\left\{A_{k m} e_{i k} e_{k i m}+A_{i m} e_{k i} e_{k m}+A_{i k} e_{k i} e_{m k}\right\} \equiv 0 \\
& {[k, m] \equiv e_{k m}\left\{A_{m i} e_{k i} e_{l k}+A_{m k} e_{i k} e_{l k}+A_{m l} e_{i k} e_{l k}\right\}} \\
& \quad+e_{m k}\left\{A_{k l} e_{i k} e_{k l}+A_{i l} e_{k i} e_{k l}+A_{i k} e_{k i} e_{l k}\right\} \equiv 0 .
\end{aligned}
$$

Das Bildungsgesetz der linken Seiten von $[k, i],[k, l],[k, m]$ liegt auf der Hand.

1) Haben beide Tetraeder eine entsprechende Ecke gemein, etwa die $i$ te, so ist $e_{i l}=e_{i l}=e_{i m}=0$ und es verschwinden $A_{i k}, A_{i l}, A_{i m}$; in der Tat treffen sich ja je zwei entsprechende Kanten: $\left(A_{i} A_{k}\right),\left(E_{i} E_{k}\right) ;\left(A_{i} A_{l}\right),\left(E_{i} E_{l}\right) ;\left(A_{i} A_{m}\right),\left(E_{i} E_{m}\right)$ in $A_{i}=E_{i}$. Die erste Identität (19) verliert dann ihre Bedeutung, da ihre Glieder einzeln verschwinden. Zwischen $A_{l m}, A_{k m}, A_{l k}$ besteht keine lineare Identitat; in der Tat hat man es mit zwei Dreiecken $\left(A_{k}, A_{l}, A_{m}\right),\left(E_{k}, E_{l}, E_{m}^{\prime}\right)$ im Raume zu tun, von denen sehr wohl zweimal entsprechende Kanten sich treffen können, ohne daß es mit dem dxitten Paare der Fall ist.

2) Die genauere Bezeichnung für $R$ wïlre $R^{(i)}$. 
Zugleich erkennt man, daß bei geeigneter Modifikation der vorgenommenen Eliminationsoperationen noch drei weitere Typen der Identität entstehen : $[l, i] \equiv[l, k] \equiv[l, m]=0 ;[m, i] \equiv[m, k] \equiv$ $[m, l]=0 ;[i, l] \equiv[i, l] \equiv[l, m]=0$.

Die somit resultierenden vier formal verschiedenen Typen der in Rede stehenden Identität lassen sich etwa in folgender Anordnung zusammenstellen:

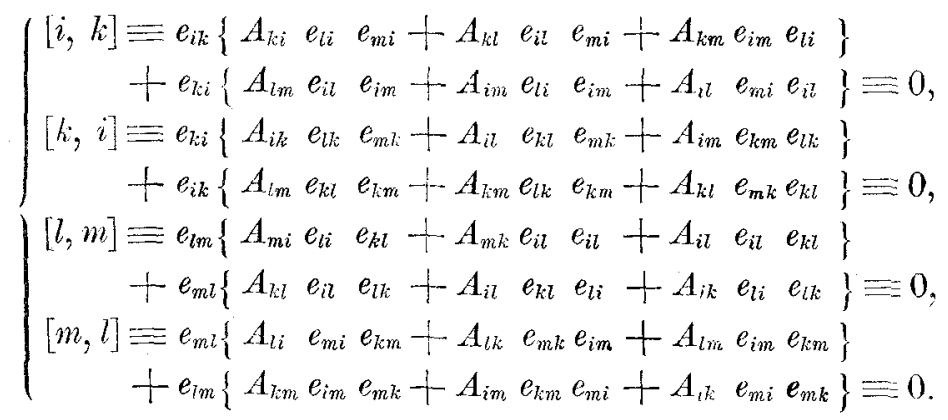

Aus diesem Schema heraus, in Verbindung mit den Werten (17) der Ausdrücke $A_{i k}$ etc. lassen sich auch leicht die besonderen Fälle diskutieren, wenn einige der $e$ verschwinden. Es ist ohne Schwierigkeit zu ersehen, daß, vorausgesetzt, dab alle $A$ verschwinden, mit irgend einem der $e$ immer zugleich mehrere derselben verschwinden.

Dabei würde das Verschwinden von $e_{i k}$ z. B. geometrisch aussagen, daß der Punkt $E_{i}$ auf der Ebene $a_{k}$ von $\mathrm{T}$ liegt, oder auch, für die ursprünglichen Tetraeder $\mathrm{T}$ und $\Delta$, daß die Ebenen $\alpha_{k}$ und $\delta_{k}\left(D_{i}, D_{l}, D_{m}\right)$ konjugiert sind. Indem wir solche besonderen Fälle beiseite lassen, sprechen wir den Inhalt der Identität (II) in dem Satze aus:

III. "Werden die Eckpunkte und damit die Kanten zweier Vierecke im Raume einander zugeordnet (von denen das eineauch einebenes sein kann) und treffen sich fünfentsprechendeKanten, so treffen sich auch die sechsten. Desgleichen, sind fünf entsprechende Kanten konjugiert in bezugauf irgend eine gegebene Fläche zweiter Klasse, so auch die sechsten."

Aber auch die beiden Relationen (19) erlauben eine einfache geometrische Bedeutung.

Denkt man sich die Ecken $E_{k}, E_{l}, E_{m}$ von $A_{i}$ aus auf die Ebene $\alpha_{i}$ projiziert und die Projektionen mit $E_{k}^{(i)}, E_{l}^{(i)}, E_{m}^{(i)}$ bezeichnet, so sind die Koordinaten $x_{k}, x_{l}, x_{m}$ derselben resp. angegeben durch die Größen $e_{k k}, e_{k l}, e_{k m} ; e_{l k}, e_{l l}, e_{l m} ; e_{m k}, e_{m l}, e_{m m}$. Verbindet man diese Punkte $E_{k}^{(i)}, E_{i}^{(i)}, E_{m}^{(i)}$ resp. mit den Punkten 
$A_{k}, A_{l}, A_{m}$, so sind die Gleichungen der Verbindungsgeraden (in der Ebene $\alpha_{i}$ ):

(20) $x_{l} e_{k m}-x_{m} e_{k l}=0, x_{m} e_{l k}-x_{k} e_{l m}=0, x_{k} e_{m l}-x_{l} e_{m l l}=0$.

Aber die notwendige und hinreichende Bedingung dafür, daß die drei Geraden (20) sich in einem Punkte treffen, wird eben durch das Verschwinden von $R$ (18) geliefert.

Die beiden Dreicke $\left(A_{k}, A_{l}, A_{m}\right)$ und $\left(E_{k}^{(i)}, E_{l}^{(i)}, E_{m}^{(i)}\right)$ befinden sich dann in perspektiver Lage; das nämliche ${ }^{1}$ ) gilt also von den beiden Dreikanten, die jene Dreiecke von der Ecke $A_{i}$ aus projizieren, d. h. die drei Ebenen, die je zwei zugeordnete Kanten verbinden, schneiden sich in einer und derselben Geraden, und die drei Geraden, in denen sich je zwei zugeordnete Ebenen schneiden,' liegen in einer und derselben Ebene.

Demnach sagt die zweite Relation (19) aus: ${ }^{2}$ )

IV. "Treffen sich je zwei zugeordnete Kanten zweier Dreieckeim Ranme (die nicht derselben Ebene angehören), so werden diebeiden Dreieckeronjedem Raumpunkte aus durch zwei Dreikante in perspektiver Lage projiziert. Umgekehrt, werden zweiDreiecke im Raume von irgend einem Raumpunkte aus durch zwei Dreikantein perspektiverLage projiziert, und treffen sich zweimal zwei zugeordnete Kanten der Dreiecke, so auch dieletzten, und die beiden Dreieckewerden von jedem Raumpunkte aus durch zwei Dreikante in perspektiver Lage projiziert."

Der hiezu dualistische Satz entspricht genau der ersten Relation (19).

Bisher war das eine der beiden Vierecke T und $\left(E_{i}, E_{k}, E_{l}\right.$, $E_{m}$ ), nämlich dasjenige, T, dessen Ecken sicher nicht in einer Ebene liegen sollten, als Koordinatentetraeder gewählt worden. Nunmehr soll die (II) entsprechende Identität für zwei beliebige Vierecke A $\left(A_{i}, A_{k}, A_{l}, A_{m}\right)$ und $\mathrm{B}\left(B_{i}, B_{k}, B_{l}, B_{m}\right)$, von denen das eine auch ein ebenes sein darf, aufgestellt werden.

Geometrisch ist klar, daf die frühere Bedingung $A_{i k}=0$, da sie jetzt aussagt, daß sich die zugeordneten Kanten $\left(A_{i}, A_{k}\right)$, $\left(B_{i}, B_{k}\right)$ treffen sollen, übergeht in:

$$
\{i, k\} \equiv\{k, i\} \equiv\left|\begin{array}{llll}
a_{i i} & a_{i k} & a_{i l} & a_{i m} \\
a_{k i} & a_{k k} & a_{k l} & a_{k m} \\
b_{i i} & b_{i k} & b_{i l} & b_{i m} \\
b_{k i} & b_{k k} & b_{k l} & b_{k m}
\end{array}\right|=0
$$

1) Der Ausdruck "Zwei Dreikante befinden sich in perspektiver Lage, wenn" ist der Kürze halber von dem entsprechenden Verhalten zweier Dreiecke in einer Ebene übertragen worden, wenn er auch sprachlich Bedenken verursacht.

2) Das Treffen zweier Kanten kann hier wiederum überall durch das Konjugiertsein derselben in bezug auf eine Fläche zweiter Klasse ersetzt werden. 
und desgleichen, daß die frühere Bedingung $e_{i k}=0$, da sie jetzt aussagt, da 3 der Punkt $B_{i}$ auf der Ebene $\alpha_{k}\left(A_{i}, A_{l}, A_{m}\right)$ liegen soll, übergeht in :

$(22) \varepsilon_{i k}=\left|\begin{array}{cccc}b_{i i} & b_{i k} & b_{i l} & b_{i m} \\ a_{i i} & a_{i k} & a_{i l} & a_{i m} \\ a_{l i} & a_{l k} & a_{l l} & a_{l m} \\ a_{m i} & a_{m k} & a_{m l} & a_{m m}\end{array}\right| \equiv b_{i i} \alpha_{k i}+b_{i k} \alpha_{l k}+b_{i l} \alpha_{k l}+b_{i m} \alpha_{k m}=0$,

wenn $\alpha_{i k}$ die erste Minore von $a_{i k}$ in der Determinante $A=\left|a_{i k}\right|$ bedeutet.

Somit sind in der obigen speziellen Identität (II) die $A_{i k}, \epsilon_{i k}$ nunmehr zu ersetzen durch Ausdrücke, die den $\left\{i, l_{i}\right\}, \Xi_{i k}$ in $(21$, 22) proportional sind, und es handelt sich nur noch um die Bestimmung dieser Proportionalitätsfaktoren. Man gelangt dazu vermöge einer linearen Koordinatentransformation, die das eine der beiden Vierecke, A - das nicht in ein ebenes ausarten sollte zum Koordinatentetraeder macht.

Seien die Punkte $A_{i}, B_{i}$ auf ein ursprüngliches Koordinatensystem ( $\xi$ ) bezogen, so setze man, wenn das neue System mit $(x)$ bezeichnet wird:

$$
\xi_{r}=a_{i r} x_{i}+a_{k r} x_{k}+a_{l r} x_{l}+a_{m r} x_{m}(r=i, k, l, m) .
$$

Dadurch gehen in der Tat die Punkte $A_{i}$ ïber in die Ecken des neuen Koordinatentetraeders der $x$. Die (23) inverse Transformation lautet:

$$
x_{r}=\alpha_{r i} \xi_{i}+\alpha_{, k} \xi_{k}+\alpha_{, i} \xi_{l}+\alpha_{r m} \xi_{m}(r=i, k, i, m) .
$$

Gehen also vermöge (23) oder auch (24) die alten Punkte $B_{i}$. ïber in die neuen Punkte $E_{i}$, so ist gemäß (24):

$$
e_{s r}=\alpha_{r i} b_{s i}+\alpha_{r k} b_{s k}+\alpha_{r l} b_{s l}+\alpha_{r m} b_{s m}(r, s=i, k, l, m) \text {. }
$$

Dann gehen aber, zufolge einfacher Determinantensätze, die früheren Ausdrücke $A_{i k}$ (17), bis auf den Faktor $A=\left|a_{i k}\right|$, über in die Ausdrücke $\{i, k\}(21)$ :

$$
A_{i k}=A \cdot\{i, k\}
$$

während die früheren Größen $e_{i k}$ direkt $z u$ den Größen $\varepsilon_{i k}(22)$ werden. Es gilt somit der Satz:

V. "Man hat nur in irgend einer der Identitäten (II) die $A_{i k}, e_{i k}$ resp. durch die in $(21,22)$ angegebenen $\{i, k\}, \varepsilon_{i k}$ zu ersetzen, um die entsprechende Identität für zwei beliebige Vierecke $\mathrm{A}\left(A_{i}\right)$ und $\mathrm{B}\left(B_{i}\right)$ zu erhalten."

Rein algebraisch lautet der Satz:

"Liegen zwei vierreihige Determinanten $\left|a_{i k}\right|,\left|b_{i k}\right|$ vor, von denen "eine, etwa die erste, nicht verschwinden soll, und bildet 
man (in dieser Reihenfolge) je aus der $i^{\text {ten }}$ und $k^{\text {ten }}$ Vertikale (oder Reihe) der ersten und der $i^{\text {ten }}$ und $k^{\text {ten }}$ Vertikale (oder Reihe) der zweiten eine neue vierreihige Determinante $\{i, k\}$, so sind diese sechs Determinanten $\{i, k\}$ an die lineare Identität des Satzes V gebunden, so daß - wenn man das Verschwinden gewisser der $\varepsilon_{i k}$ ausschließt - das Verschwinden von irgend fünf der sechs Determinanten dasjenige der letzten bedingt."

Der durch Satz $V$ charakterisierten Identität läßt sich eine symmetrische Gestalt geben, wenn man mit Rücksicht auf die geometrische Bedeutung von $\varepsilon_{i k}=0$, oder was auf dasselbe hinauskommt, auf die in (22) angegebene Darstellung:

$$
\varepsilon_{i k}=b_{i i} \alpha_{k i}+b_{i k} \alpha_{k k}+b_{i l} \alpha_{k l}+b_{i m} \alpha_{k m} \equiv\left(B_{i}, \alpha_{k}\right),
$$

wo die $\alpha_{k i}, a_{k k}, \alpha_{k l}, \alpha_{k m}$ geometriseh die Koordinaten der Ebene $\alpha_{k}\left(A_{i}, A_{l}, A_{m}\right)$ sind, von vornherein die beiden Vierecke $\mathbf{A}$ und $\mathbf{B}$ dualistisch gegenüberstellt.

Danach denke man sich das Viereck A durch die Koordinaten $\alpha_{i r}(i, r=i, k, l, m)$ seiner Ebenen, das Viereck $\mathrm{B}$, wie oben durch die Koordinaten $b_{i r}(i, r=i, k, l, m)$ seiner Ecken gegeben. Dann erscheint $\varepsilon_{i k}(22)$ als bilineare Invariante $\left(B_{i}, \alpha_{k}\right)$ der beiden Linearformen, deren Verschwinden den Punkt $B_{i}$ resp. die Ebene $\alpha$ darstellt. Anderseits wird der Ausdruck $\{i, k\}$ (21), wenn man die Strahlenkoordinaten $p_{r s}^{(i k)}=\left|\begin{array}{ll}a_{i r} & a_{i s} \\ a_{k r} & a_{k s}\end{array}\right|, \quad q_{t a t}^{(i k)}=\left|\begin{array}{ll}b_{i t} & b_{i u} \\ b_{k t} & b_{k u}\end{array}\right|$ der Kanten $\left(A_{i}, A_{k}\right),\left(B_{i}, B_{k}\right)$ einführt, die bilineare Invariante der beiden speziellen linearen Komplexe jener Kanten:

$$
\{i, k\}=\Sigma \Sigma p_{i s}^{(i k)} q_{t u}^{(i k)} \quad(r, s, t, u \text { zyklisch }=i, k, l, m) .
$$

Bedient man sich nunmehr der Achsenkoordinaten der Kante $\left(A_{i}, A_{k}\right)=\left(\alpha_{l}, \alpha_{m}\right)$, nämlich der Größjen $\pi_{t u}^{(l m)}=\left|\begin{array}{ll}\alpha_{l t} & \alpha_{l u} \\ \alpha_{m t} & \alpha_{m u}\end{array}\right|$, so nimmt $\{i, k\}$ die Gestalt an:

$$
\{i, k\}=\Sigma \Sigma \pi_{t u}^{(l m)} q_{i u}^{(i k)} .
$$

Demnach gilt als Modifikation des Satzes V:

$V^{\prime}$. "Denkt man sich von den beiden Vierecken $A$ und $B$ "das eine, B, durch die Koordinaten seiner Ecken, das andere, A, durch die Koordinaten seiner Ebenendargestellt, und ersetzt demgemäB in der zwischen den Kanten $z$ weier Tetraeder bestehenden speziellen Identität (II) die $A_{i k}$ durch die Ausdrücke $\{i, k\}\left(21^{\prime \prime}\right)$, und die $e_{i k}$ durch die Ausdrücke $\varepsilon_{i k}\left(22^{\prime}\right)$, so nimmt die Identität eine für beide Vierecke A, B symmetrische Gestalt an."

Endlich kann auf Grund dieses Satzes die Identität (II) auch malogeometrisch gedeutet werden. Denkt man sich alles auf ein 
ursprüngliches rechtwinkliges Koordinatensystem bezogen, und zwar die Gleichungen der Ebenen $\alpha_{k}$ von $A$ in der Hesse schen Normalform gegeben, so werden die Größen $\varepsilon_{i k}\left(22^{\prime}\right)$ die Längen der Lote, die man von Ecken $B_{i}$ auf die Ebenen $\alpha_{k}$ fällen kann, und die Ausdrücke $\{i, k\}\left(21^{\prime \prime}\right)$ die $\mathrm{M} \circ \mathrm{m} \in \mathrm{n}$ te je zweier zugeordneter Kanten $\left(A_{i}, A_{k}\right),\left(B_{i}, B_{k}\right)$.

Die Lote sind natürlich auch ersetzbar durch die anderen, die man von den Ecken $A_{i}$ auf die Ebenen $\beta_{k}$ von B fällen kann.

In dem besonderen Falle, daß das Viereck $B$ in ein ebenes ausartet, wird, wenn unter $\eta_{i k}$ der zu $\left(22^{\prime}\right)$ analoge Ausdruck $\left(A_{i}, \beta_{k}\right)$ verstanden wird, da jetzt alle vier Ebenen $\beta_{k}$ in eine einzige zusammenfallen,

$$
\eta_{i k}=\eta_{i l}=\eta_{i m}=\eta_{i} ; \text { etc. }
$$

und die Identität (II) nimmt die einfache Gestalt an:

$$
\Sigma\{i, k\} \eta_{l} \eta_{m}=0
$$

In dem obigen Beweise der auf zwei beliebige Vierecke A, B ausgedehnten Identität (II) war vorausgesetzt, daß wenigstens das eine der beiden, etwa $\mathrm{A}$, nicht in ein ebenes ausarten solle. Da aber die Identität für im übrigen willkürliche Werte der Koordinaten beider Punktquadrupel $\mathrm{A}, \mathrm{B}$ erfüllt ist, so muß sie, nach einem algebraischen Grundsatze, bestehen bleiben, wenn auch beide Vierecke je in einer Ebene liegen, da dann weder die $\{i, k\}$ (21) noch die $\varepsilon_{i k}(22)$ einzeln verschwinden; ausgeschlossen bleibt allein der Fall (s. u.), dab auch die Ebenen beider Vierecke zusammenfallen, da dies das Verschwinden sämtlicher $\{i, k\}$ nach sich ziehen würde.

Es ist aber nützlich, auch für zwei Vierecke, von denen eines bereits als ein ebenes angenommen wird, den Beweis der zugehörigen Identität so zu führen, daß den Koordinaten der Ecken des zweiten Viereckes keine Beschränkung hinsichtlich des etwaigen Verschwindens ihrer Determinante auferlegt wird.

Es seien die Ecken des ebenen Viereckes A in der Ebene $x_{m}=0$ gelegen, und zwăr $A_{i}, A_{k}, A_{l}$ als Koordinatenecken, während die vierte Ecke durch die Koordinaten $\lambda_{i}, \lambda_{k}, \lambda_{l}, 0$ festgelegt sei; die Koordinaten der Ecken $B_{i}$ von $\mathrm{B}$ seien wie bisher mit $b_{i i}, b_{i k}$, $b_{i l}, b_{i m}$ bezeichnet.

Bedient man sich noch der Abkürzung $b_{r m}=b_{r}(r=i, k, l, m)$, so berechnen sich gemäß (2i), die sechs Ausdrücke $\{i, k\}$ zu:

$$
\left\{\begin{array}{l}
\{i, k\}=+b_{i l} b_{k}-b_{k l} b_{i} \\
\{k, l\}=-b_{l i} b_{k} \quad+b_{k i} b_{l} \\
\{l, i\}=b_{l k} b_{i}-b_{i k} b_{l} \\
\{i, m\}=\lambda_{k}\left(b_{i l} b_{m}-b_{m l} b_{i}\right)-\lambda_{l}\left(b_{i k} b_{m}-b_{m k} b_{i}\right) \\
\{k, m\}=\lambda_{l}\left(b_{k i} b_{m}-b_{m i} b_{l}\right)-\lambda_{i}\left(b_{k l} b_{m}-b_{m l} b_{k}\right) \\
\{l, m\}=\lambda_{i}\left(b_{l k} b_{m}-b_{m k} b_{l}\right)-\lambda_{k}\left(b_{l i} b_{m}-b_{m i} b_{l}\right)
\end{array}\right.
$$


Weiter erhält man, gemäß (22), für die $\varepsilon_{l k}$ die Werte:

$$
\left\{\begin{aligned}
& \varepsilon_{i k}=\lambda_{k} b_{i}, \quad \varepsilon_{i l}=\lambda_{l} b_{i}, \quad \varepsilon_{i k}=-b_{i}, \\
& \varepsilon_{k i} b_{k}, \varepsilon_{k l}=\lambda_{l} b_{k}, \quad \varepsilon_{k m}=-b_{k}, \\
& \varepsilon_{l i}=\lambda_{l}, \quad \varepsilon_{k}=\lambda_{k} b_{l}, \varepsilon_{l m}=-b_{l}, \\
& \varepsilon_{m i}=\lambda_{i} b_{m}, \quad \varepsilon_{m l}=\lambda_{k} b_{m}, \quad \varepsilon_{m l}=\lambda_{l} b_{m} .
\end{aligned}\right.
$$

Damit geht für den vorliegenden Fall die linke Seite der Identität (II), etwa nach dem Typus $[i, k]$, abgesehen von dem Faktor $\lambda_{i} b_{i}$, über in $\left.{ }^{1}\right)$ :

$$
\begin{aligned}
{[i, k] \equiv } & \lambda_{k}\left[\{k, i\} \lambda_{i} b_{l} b_{m}+\{k, l\} \lambda_{l} b_{i} b_{m}-\{k, m\} b_{i} b_{l}\right] \\
& +b_{k}\left\{-\{l, m\} \lambda_{l} b_{i}-\{i, m\} \lambda_{i} b_{l}+\{i, l\} \lambda_{i} \lambda_{l} b_{m}\right]
\end{aligned}
$$

wo für die $\{k, i\}$ etc. die Werte (28) zu substituieren sind.

Aus der ersten eckigen Klammer rechts, die in $\lambda_{k}$ multipliziert ist, läßt sich noch der Faktor $b_{k}$ herauszieben; der andere Faktor wird

$$
b_{l} \lambda_{i}\left(b_{i l} b_{m}-b_{m l} b_{l}\right)-b_{i} \lambda_{l}\left(b_{l i} b_{m}-b_{m i} b_{l}\right)
$$

Aus der zweiten eckigen Klammer, die in $b_{k}$ multipliziert ist, läßt sich noch der Faktor $\lambda_{k}$ herausziehen; der verbleibende Faktor ist dann genau das Negative des Ausdruckes (31).

Damit ist in der Tat das identische Verschwinden der Simultaninvariante $[i, k](21)$ eines ebenen Viereckes $A$ und eines beliebigen Vierecks $B$ direkt nachgewiesen.

Wie oben betont, blieb bisher nur der Fall ausgeschlossen, daß das Viereck $B$ so in ein ebenes ausartet, daß die Ebenen beider Vierecke zusammenfallen, da dann die Glieder des Audruckes $[i, k]$ einzeln verschwinden. Nun ist es aber gerade ein Fundamentalsatz bei v. Staudt, ${ }^{2}$ ) - der ibm dazu dient, den Begriff des Harmonischen unabhängig von Maßbegriffen zu entwickeln - daß für zwei ebene Vierecke in derselben Ebene die Eigenschaft besteht: "Ordnet man die Punkte und damit auch die Kanten (Seiten) zweier Vierecke in derselben Ebene einander zu und liegen die Schnittpunkte von fünf Paaren entsprechender Kanten auf ein und derselben Geraden, so gebört dieser auch der Sehnittpunkt des sechsten Paares an."

Diese Eigenschaft zweier Vierecke in ein und derselben Ebene läßt sich aus der entsprechenden zweier ebener Vierecke in verschiedenen Ebenen nach v. Staudt direkt durch einen Grenzproze $\$$ herleiten, indem man etwa die eine der beiden Ebenen um ihre Schnittachse mit der anderen so lange dreht, bis sie mit der letzteren zusammenfällt.

1) Legt man den Koordinaten $\lambda_{i}, \lambda_{k}, \lambda_{l}, 0$ der vierten Ecke von A, was erlaubt ist, die kanonischen Werte $-1,-1,-1,0$ bei, so vereinfacht sich der Ausdruck $[i, k](30)$ zu: $\Sigma\{i, k\} b_{l} b_{m}$, in Übereinstimmung mit (II').

2) Geometrie der Lage, p. 40. 
Um so bemerkenswerter scheint es, dah die für zwei ebene Vierecke in derselben Ebene gültige Identität, die der algebraische Ausdruck des von Staudtschen Satzes ist, von wesentlich anderem Charakter ist, als die von uns oben für zwei Vierecke im Raume aufgestellte. Wir kommen gleich darauf zurück.

Verfolgt man die für den Fall der Ebene gültige Identität weiter, so gelangt man $\mathrm{zu}$ folgendem geometrischen Kriterium für zwei Vierecke, bei denen der $v$. Sta ud sche Satz eintritt:

„Damit zwei punktweise einander zugeordnete Vierecke in ein und derselben Ebene in der v. Staudtschen Beziehung stehen, dab sich fünf Paare entsprechender Kanten beider Vierecke - und damit auch das sechste Paar - auf ein und derselben Geraden begegnen, ist notwendig und hinreichend: erstens, dab sich beide Vierecke - ganz wie im Raume - in perspektiver Lage befinden; zweitens überdies, dabsich auch die beiden Hauptdreiecke ${ }^{1}$ ) der Vierecke in perspektiver Lage befinden, und zwar so, dab ihr Perspektivitätszentrum mit dem der beiden Vierecke zusammenfällt."

Diese Entwicklungen für den Fall der Ebene habe ich gleichfalls vollständig durchgeführt und werde darauf an anderer Stelle eingehen. Hier sei nur auf folgende wesentliche Gesichtspunkte hingewiesen.

Der oben erwähnte Grenzprozeß, vermöge dessen die beiden ursprünglich verschiedenen Ebenen zweier Vierecke koinzidieren, läßt sich noch in mannigfaltiger Weise variieren. Es sind aber dabei zwei wesentlich verschiedene Hauptfälle zu unterscheiden.

Entweder bleibt bei der Koinzidenz der beiden Ebenen deren Achse eine völlig bestimmte Gerade, oder aber diese Achse wird (in der Grenzebene) völlig unbestimmt.

Im ersteren Falle kann man etwa die beiden Ebenen durch die Gleichungen $E=0$ und $E+\varepsilon H=0$ darstellen, und dann, bei festgehaltenen $E, H$, den Parameter $\varepsilon$ gegen Null konvergieren lassen. Dann läßt sich der Grenzprozeß an der Identität (II) so ausführen, daß der Faktor $\varepsilon$ vor die linke Seite der Identität tritt, und diese somit im Grenzfalle völlig erhalten bleibt. Dann gelangt man direkt zum v. Staudtschen Satze für zwei ebene Vierecke.

1) Der innere Grund dieser Erscheinung tritt hervor, wenn man den leicht beweisbaren Satz benützt, daß zwei perspektive Vierecke einer Ebene $E$ stets and zwar noch auf $\infty^{4}$ Arten - als Schnitt eines vollständigen räumlichen Sechseckes mit der Ebene $E$ betrachtet werden können. Ist $O$ das Perspektivitätszentrum, so hat man nur zwei Raumpunkte 1, 2 beliebig, aber so zu wählen, daß ihre Kante durch $O$ hindurchgeht; dann sind die vier übrigen Ecken 3, 4, 5, 6 eindeutig bestimmt.

Die notwendige und hinreichende Bedingung für die v. Staudtsche Lage beider ebenen Vierecke ist dann die, da 3 die vier Raumpunkte 3, 4, 5, 6 einer und derselben Ebene angehören. Dann wird die angegebene Eigenschaft der beiden Hauptdreiecke eine angenscheinliche. 
Im zweiten Falle dagegen, wo die Achse der beiden Ebenen $E, H$ im Grenzfalle innerbalb der Ebene $E$ ganz unbestimmt wird, versagt die Identität (II) in der Tat. Denn jetzt ist die geometrische Fragestellung für die Figur der beiden ebenen Vierecke eine ganz andere geworden. Bezeichnet man wieder die zugeordneten Seiten beider Vierecke mit $1,2, \ldots 6 ; 1^{\prime}, 2{ }^{\prime}, \ldots 6$, so verbinde man die Schnittpunkte irgend zweier Paare, z. B. $\left(1,1^{\prime}\right),\left(2,2^{\prime}\right)$ durch eine Gerade $g$. Sodann stelle man sukzessive die Bedingungen auf, daß die Schnittpunkte $\left(3,3^{\prime}\right),\left(4,4^{\prime}\right),\left(5,5^{\prime}\right),\left(6,6^{\prime}\right)$ auf $g$ liegen : diese Bedingungen seien mit $[123]=0,[124]=0,[125]=0,[126]=0$ bezeichnet. Dann muf von diesen vier Bedingungen je eine eine Folge der drei übrigen sein, d. h. zwischen den vier linken Seiten findet nunmehr eine lineare Identität statt, die jetzt das Äquivalent des $\nabla$. Staudtschen Satzes wird. Die weitere Entwicklung lehrt dann, daß die neue Fragestellung weit tiefer in die Natur der Konfiguration führt als die ursprüngliche.

Die Identität (II) läßt sich, geometrisch wie algebraisch nach verschiedenen Richtungen ausdebnen. Die Mannigfaltigkeit der auftretenden Beziehungen wird indessen gleich eine so große, daß der Gegenstand an Interesse merklich verliert. ${ }^{1}$ ).

Indem wir zu dem eingangs dieses Paragraphen aufgestellten Satze über die beiden Vierecke $T$ und $\Delta$ zurückkehren, bemerken wir, daß, wie in $\$ 1$, der geführte Beweis gültig bleibt, wenn die zu Gerade gelegte Fläche zweiter Klasse $\Gamma$ (1) ersetzt wird durch eine beliebige Ebenenkorrelation:

$$
\Sigma \Sigma u_{i} v_{k} c_{i k}=0\left(c_{i k} \geqq c_{k i}\right) \text {. }
$$

Stehen demnach zwei Tetraeder $\mathrm{T}\left(A_{i}\right)$ und $H\left(Y_{i}\right)$ in der besonderen Beziehung, daß sich die durch die Ecken $Y_{i}$ von $H$ gehenden, zu den entsprochenden Ebenen $\alpha_{i}$ von $T$ bezüglich der Korrelation (32) konjugierten ${ }^{2}$ ) Geraden $p_{i}$ in einem Punkte $P(y)$ treffen, so treffen sich auch die durch die Ecken $A_{i}$ von 'T gehenden, zu den entsprechenden Ebenen $v_{i}$ von $H$ konjugierten Geraden $g_{i}$ in einem Punkte $Q(z)$, und die reziprok entsprechenden Kanten beider Tetraeder sind je zu einander konjugiert.

Die Beziehung der beiden Punkte $P(y), Q(z)$ soll algebraisch dargestellt werden.

Bedeutet wieder $\left(C_{i}, C_{k}, C_{l}, C_{n n}\right)$ das Poltetraeder $\left.{ }^{2}\right)$ von $\mathrm{T}$ bezüglich (32), so muß die Gerade $P Y_{r}(r=i, k, l, m)$ je durch den

1) Liegen z. B. zwei fünfreihige Determinanten vor und man bildet jeweils unter Weglassung einer Reihe, aus der $i^{\text {ten }}$ und $k^{\text {ten }}$ Kolonne beider verbleibenden Matrices eine vierreihige Determinante, so ist das Verschwinden aller dieser 50 Determinanten aquivalent mit 20 Bedingungen, von denen wiederum 9 eine Folge der übrigen 11 sind. Geometrisch sagen diese Bedingungen aus, daß zwei Fünfecke im $R_{4}$ perspektiv liegen.

2) Es wird kein Mißverständnis veranlassen, wenn der Kürze halber die Worte "konjugiert, $\mathrm{PoI}^{\text {" }}$ von der ursprünglichen Polarität (1) auf die entsprechenden Begriffe bei der Korrelation (32) übertragen werden. 
Punkt $C_{r}$ gehen, d. h. es müssen, in Verallgemeinerung der Formeln (2), Relationen bestehen von der Form:

$$
Y_{r s}=\lambda_{r} y_{s}-\mu_{r} c_{r s}(r, s=i, k, l, m) .
$$

Um den dem Punkte $P(y)$ zugeordneten Punkt $Q(z)$ zu bestimmen, dient eine völlig analoge Rechnung, wie die zu Beginn von $\S 1$ durchgeführte, und man erhält in Verallgemeinerung der Formeln (I) daselbst:

$$
z_{i}: z_{k}: z_{l}: z_{m}=\frac{\lambda_{i}}{\mu_{i}}: \frac{\lambda_{k}}{\mu_{k}}: \frac{\lambda_{l}}{\mu_{l}}: \frac{\lambda_{m}}{\mu_{m}}
$$

d. h. die Koordinaten von $Q(z)$ sind den nicht homogenen Parametern $\frac{\lambda}{\mu}$ der Punkte $Y_{r}$ auf den Geraden $p_{r}$, die den Punkt $P(y)$ mit den vier Punkten $C_{r}(r=i, k, l, m)$ verbinden, proportional.

Soll insbesondere der Punkt $Q(z)$ ein beliebig vorgegebener sein, so sind die $\frac{\lambda_{r}}{\mu_{r}}$ bis auf einen willkürlichen Faktor $\rho$ bestimmt; es gehören also noch $\infty^{1}$ Punktequadrupel $Y_{r}$ daza, die auf den Geraden $p_{r}$ projektive Punktreihen beschreiben. Speziell kann man den Punkt $Q(z)$ mit dem in $\$ 1$ bestimmten zusammenfallen lassen oder auch mit dem Ausgangspunkte $P(y)$ selbst.

Des weiteren läßt sich die Auffassung der Figur variieren, wie in $\S 1$; man nehme das Tetraeder T, den Punkt $P(y)$ und das Tetraeder $\mathrm{H}$ der Punkte $Y_{r}$ als beliebig gegeben an und suche die zugehörigen Korrelationen (32). Die Koeffizienten $c_{r s}$ in (32) erscheinen jetzt als binäre Linearformen der $Y_{r s}$ and $y_{s}$ :

$$
c_{r s}=l_{r} y_{s}+m_{r} Y_{r s}(r, s=i, k, l, m),
$$

so daß die bezüglichen Korrelationen (32) noch eine $\infty^{7}$ lineare Schar bilden mit den acht homogenen Parametern $l_{r}, m_{r}$.

Soll eine derartige Korrelation (32) insbesondere zu einem Polarsystem $\Gamma(1)$ werden, also stets $c_{i l}=c_{k i}$ sein, so involviert das sechs lineare homogene Relationen zwischen den acht Größen $l_{r}, m_{r}$; jene Bedingungen sind linear unabhängig, denn mittels elementarer Rechnung lassen sich die $c_{i k}$ als binäre Linearformen etwa von $l_{1}$ und $m_{1}$ berechnen.

Zu irgend einem Tetraeder $H$ gehört also, ganz wie in $\S 1$, zu einem, T einbeschriebenen, noch eine $\infty^{1}$ lineare Schar von Flächen zweiter Klasse $\Gamma$ mit entsprechenden Eigenschaften wie dort; der Punkt $Q(z)$ beschreibt wieder eine Gerade etc.

Die Formeln des $\S 1$ gehen durch Spezialisierung aus den jetzigen (33) hervor, wenn man $\lambda_{r}$ proportional mit $c_{r r}, \mu_{r}$ proportional mit $y_{r}$ wählt. Soll der Punkt $Q(z)$ nebst der Fläche $\Gamma$ 
unverändert bleiben, so lassen sich immer noch die Quotienten $\frac{\lambda_{r}}{\mu_{r}}=\frac{c_{r r}}{y_{r}}$ mit einem beliebigen Faktor $\rho$ versehen, d. h. die Formeln (2) lassen sich ersetzen durch:

$$
Y_{r s}=p c_{r r} y_{s}-y_{r} c_{r s}
$$

wie im aligemeinen Falle tritt also an Stelle des einen Tetraeders $\mathrm{H}$ die $\infty^{1}$ Schar, deren Ecken auf den dortigen Geraden $p$ projektive Punktreihen beschreiben. 